\title{
Global Dynamics of Virus Infection Model with Antibody Immune Response and Distributed Delays
}

\author{
A. M. Elaiw, ${ }^{1,2}$ A. Alhejelan, ${ }^{1,3}$ and M. A. Alghamdi ${ }^{1}$ \\ ${ }^{1}$ Department of Mathematics, Faculty of Science, King Abdulaziz University, P.O. Box 80203, Jeddah 21589, Saudi Arabia \\ ${ }^{2}$ Department of Mathematics, Faculty of Science, Al-Azhar University, Assiut, Egypt \\ ${ }^{3}$ Department of Mathematics, Faculty of Arts and Science, Qassim University, Buraidah 71511, Saudi Arabia
}

Correspondence should be addressed to A. M. Elaiw; a_m_elaiw@yahoo.com

Received 8 August 2013; Accepted 7 October 2013

Academic Editor: Victor S. Kozyakin

Copyright (C) 2013 A. M. Elaiw et al. This is an open access article distributed under the Creative Commons Attribution License, which permits unrestricted use, distribution, and reproduction in any medium, provided the original work is properly cited.

\begin{abstract}
We present qualitative behavior of virus infection model with antibody immune response. The incidence rate of infection is given by saturation functional response. Two types of distributed delays are incorporated into the model to account for the time delay between the time when uninfected cells are contacted by the virus particle and the time when emission of infectious (matures) virus particles. Using the method of Lyapunov functional, we have established that the global stability of the steady states of the model is determined by two threshold numbers, the basic reproduction number $R_{0}$ and antibody immune response reproduction number $R_{1}$. We have proven that if $R_{0} \leq 1$, then the uninfected steady state is globally asymptotically stable (GAS), if $R_{1} \leq 1<R_{0}$, then the infected steady state without antibody immune response is GAS, and if $R_{1}>1$, then the infected steady state with antibody immune response is GAS.
\end{abstract}

\section{Introduction}

In the past ten years there has been a growing interest in modeling viral infections for the study and characterization of host infection dynamics. The mathematical models, based on biological interactions, present a framework which can be used to obtain new insights into the viral dynamics and to interpret experimental data. Many authors have formulated mathematical models to describe the population dynamics of several viruses such as, human immunodeficiency virus (HIV) [1-14], hepatitis B virus (HBV) [15, 16], and hepatitis $\mathrm{C}$ virus (HCV) $[17,18]$. During viral infections, the host immune system reacts with antigen-specific immune response. In particular, B cells play a critical role in antiviral defense by attacking free virus particles by making antibodies to clear antigens circulating in blood and lymph. The antibody immune response is more effective than the cellmediated immune in some diseases like in malaria infection [19].

Mathematical models for virus dynamics with the antibody immune response have been developed in [20-26]. The basic virus dynamics model with antibody immune response was introduced by Murase et al. [21] as

$$
\begin{aligned}
\dot{x}(t) & =\lambda-d x(t)-\beta x(t) v(t), \\
\dot{y}(t) & =\beta x(t) v(t)-\delta y(t), \\
\dot{v}(t) & =N \delta y(t)-c v(t)-q v(t) z(t), \\
\dot{z}(t) & =r v(t) z(t)-\mu z(t),
\end{aligned}
$$

where $x(t), y(t), v(t)$, and $z(t)$ represent the populations of uninfected cells, infected cells, virus, and $\mathrm{B}$ cells at time $t$, respectively; $\lambda$ and $d$ are the recruited rate and death rate constants of uninfected cells, respectively; $\beta$ is the infection rate constant; $N$ is the number of free virus produced during the average infected cell life span; $\delta$ is the death rate constant of infected cells; $c$ is the clearance rate constant of the virus; $r$ and $\mu$ are the recruited rate and death rate constants of B cells; and $q$ is the B cells neutralizion rate. Model (1) is based on the assumption that the infection could occur and that the viruses are produced from infected cells instantaneously, once the 
uninfected cells are contacted by the virus particles. Other accurate models incorporate the delay between the time viral entry into the uninfected cell and the time the production of new virus particles, modeled with discrete time delay or distributed time delay using functional differential equations (see e.g., [7-14, 27]). In [7-14, 27], the viral infection models are presented without taking into consideration the antibody immune response. In $[25,28]$, global stability of viral infection models with antibody immune response and discrete delays has been studied.

In model (1) the infection rate is assumed to be bilinear in $x$ and $v$ however, this bilinear incidence rate associated with the mass action principle is insufficient to describe the infection process in detail $[29,30]$. For example, a less than linear response in $v$ could occur due to saturation at high virus concentration, where the infectious fraction is high so that exposure is very likely. Thus, it is reasonable to assume that the infection rate is given by saturation functional response [31]. In [26], a virus infection model with antibody immune response and with saturation incidence rate has been considered. However, the time delay was not considered.

In this paper, we assume that the infection rate is given by saturation functional response. We incorporate two types of distributed delays into the model to account for the time delay between the time when uninfected cells are contacted by the virus particle and the time of emission of infectious (matures) virus particles. The global stability of this model is established using Lyapunov functionals, which are similar in nature to those used in [32]. We prove that the global dynamics of this model is determined by the basic reproduction number $R_{0}$ and antibody immune response reproduction number $R_{1}$. If $R_{0} \leq 1$, then the uninfected steady state is globally asymptotically stable (GAS), if $R_{1} \leq 1<R_{0}$, then the infected steady state without antibody immune response is GAS, and if $R_{1}>1$, then the infected steady state with antibody immune response is GAS.

\section{The Model}

In this section we propose a delay mathematical model of viral infection with saturation functional response which describes the interaction of the virus with uninfected and infected cells, taking into account the effect of antibody immune response. Consider

$$
\begin{aligned}
& \dot{x}(t)=\lambda-d x(t)-\frac{\beta x(t) v(t)}{1+\alpha v(t)} \\
& \dot{y}(t)=\beta \int_{0}^{h} f(\tau) e^{-m \tau} \frac{x(t-\tau) v(t-\tau)}{1+\alpha v(t-\tau)} d \tau-\delta y(t), \\
& \dot{v}(t)=N \delta \int_{0}^{\omega} g(\tau) e^{-n \tau} y(t-\tau) d \tau-c v(t)-q v(t) z(t),
\end{aligned}
$$

$$
\dot{z}(t)=r v(t) z(t)-\mu z(t)
$$

where $\alpha$ is a positive constant, and all the variables and parameters of the model have the same meanings as given in model (1). To account for the time lag between viral contacting a target cell and the production of new virus particles, two distributed intracellular delays are introduced. It is assumed that the uninfected cells that are contacted by the virus particles at time $t-\tau$ become infected cells at time $t$, where $\tau$ is a random variable with a probability distribution $f(\tau)$ over the interval $[0, h]$ and $h$ is limit superior of this delay. The factor $e^{-m \tau}$ accounts for the probability of surviving the time period of delay, where $m$ is the death rate of infected cells but not yet virus producer cells. On the other hand, it is assumed that a cell infected at time $t-\tau$ starts to yield new infectious virus at time $t$ where $\tau$ is distributed according to a probability distribution $g(\tau)$ over the interval $[0, \omega]$ and $\omega$ is limit superior of this delay. The factor $e^{-n \tau}$ accounts for the probability of surviving during the time period of delay, where $n$ is constant. All the parameters are supposed to be positive.

The probability distribution functions $f(\tau)$ and $g(\tau)$ are assumed to satisfy $f(\tau)>0$ and $g(\tau)>0$, and

$$
\begin{gathered}
\int_{0}^{h} f(\tau) d \tau=\int_{0}^{\omega} g(\tau) d \tau=1, \\
\int_{0}^{h} f(u) e^{s u} d u<\infty, \quad \int_{0}^{\omega} g(u) e^{s u} d u<\infty,
\end{gathered}
$$

where $s$ is a positive number. Then

$$
\begin{aligned}
& 0<\int_{0}^{h} f(\tau) e^{-m \tau} d \tau \leq 1, \quad \text { for } m \geq 0, \\
& 0<\int_{0}^{\omega} g(\tau) e^{-n \tau} d \tau \leq 1, \quad \text { for } n \geq 0 .
\end{aligned}
$$

Let $F=\int_{0}^{h} f(\tau) e^{-m \tau} d \tau$ and $G=\int_{0}^{\omega} g(\tau) e^{-n \tau} d \tau$. The initial conditions for system (2)-(5) take the form

$$
\begin{aligned}
x(\theta) & =\varphi_{1}(\theta), \quad y(\theta)=\varphi_{2}(\theta), \\
v(\theta) & =\varphi_{3}(\theta), \quad z(\theta)=\varphi_{4}(\theta), \\
\varphi_{j}(\theta) & \geq 0, \quad \theta \in[-\rho, 0), \quad j=1, \ldots, 4, \\
\varphi_{j}(0) & >0, \quad j=1, \ldots, 4,
\end{aligned}
$$

where $\rho=\max \{h, \omega\},\left(\varphi_{1}(\theta), \varphi_{2}(\theta), \ldots, \varphi_{4}(\theta)\right) \in C([-\rho, 0]$, $\left.\mathbb{R}_{+}^{4}\right)$, where $C\left([-\rho, 0], \mathbb{R}_{+}^{4}\right)$ is the Banach space of continuous functions mapping the interval $[-\rho, 0]$ into $\mathbb{R}_{+}^{4}$. By the fundamental theory of functional differential equations [33], system (2)-(5) has a unique solution satisfying the initial conditions (8).

2.1. Nonnegativity and Boundedness of Solutions. In the following, we establish the nonnegativity and boundedness of solutions of (2)-(5) with initial conditions (8).

Proposition 1. Let $(x(t), y(t), v(t), z(t))$ be any solution of (2)-(5) satisfying the initial conditions (8); then $x(t), y(t), v(t)$, and $z(t)$ are all nonnegative for $t \geq 0$ and ultimately bounded.

Proof. First, we prove that $x(t)>0$ for all $t \geq 0$. Assume that $x(t)$ loses its non-negativity on some local existence interval 
$[0, \ell]$ for some constant $\ell$, and let $t_{1} \in[0, \ell]$ be such that $x\left(t_{1}\right)=0$. From (2) we have $\dot{x}\left(t_{1}\right)=\lambda>0$. Hence, $x(t)>0$ for some $t \in\left(t_{1}, t_{1}+\varepsilon\right)$, where $\varepsilon>0$ is sufficiently small. This leads to contradiction, and hence $x(t)>0$ for all $t \geq 0$. Now from (3), (4), and (5) we have

$$
\begin{aligned}
y(t)= & y(0) e^{-\delta t}+\beta \int_{0}^{t} e^{-\delta(t-\eta)} \\
& \times \int_{0}^{h} f(\tau) e^{-m \tau} \frac{x(\eta-\tau) v(\eta-\tau)}{1+\alpha v(\eta-\tau)} d \tau d \eta \\
v(t)= & v(0) e^{-\int_{0}^{t}(c+q z(\xi)) d \xi} \\
& +N \delta \int_{0}^{t} e^{-\int_{\eta}^{t}(c+q z(\xi)) d \xi} \\
& \times \int_{0}^{\omega} g(\tau) e^{-n \tau} y(\eta-\tau) d \tau d \eta, \\
z(t)= & z(0) e^{-\int_{0}^{t}(\mu-r v(\xi)) d \xi}
\end{aligned}
$$

confirming that $y(t) \geq 0, v(t) \geq 0$, and $z(t) \geq 0$ for all $t \in$ $[0, \rho]$. By a recursive argument, we obtain $y(t) \geq 0, v(t) \geq 0$, and $z(t) \geq 0$ for all $t \geq 0$.

Next we show the boundedness of the solutions. From (2) we have $\dot{x}(t) \leq \lambda-d x(t)$. This implies lim $\sup _{t \rightarrow \infty} x(t) \leq \lambda / d$. Let $X(t)=\int_{0}^{h} f(\tau) e^{-m \tau} x(t-\tau) d \tau+y(t)$; then

$$
\begin{aligned}
\dot{X}(t)= & \int_{0}^{h} f(\tau) e^{-m \tau}(\lambda-d x(t-\tau) \\
& \left.-\frac{\beta x(t-\tau) v(t-\tau)}{1+\alpha v(t-\tau)}\right) d \tau \\
& +\int_{0}^{h} f(\tau) e^{-m \tau} \frac{\beta x(t-\tau) v(t-\tau)}{1+\alpha v(t-\tau)} d \tau-\delta y(t) \\
= & \lambda \int_{0}^{h} f(\tau) e^{-m \tau} d \tau-d \\
& \times \int_{0}^{h} f(\tau) e^{-m \tau} x(t-\tau) d \tau-\delta y(t) \\
\leq & \lambda \int_{0}^{h} f(\tau) e^{-m \tau} d \tau-\sigma_{1} \\
& \times\left[\int_{0}^{h} f(\tau) e^{-m \tau} x(t-\tau) d \tau+y(t)\right] \\
\leq & \lambda-\sigma_{1} X(t),
\end{aligned}
$$

where $\sigma_{1}=\min \{d, \delta\}$. Hence, $\limsup _{t \rightarrow \infty} X(t) \leq L_{1}$, where $L_{1}=\lambda / \sigma_{1}$. Since $\int_{0}^{h} f(\tau) e^{-m \tau} x(t-\tau) d \tau>0$, then $\limsup _{t \rightarrow \infty} y(t) \leq L_{1}$. On the other hand, let $Z(t)=v(t)+$ $(q / r) z(t)$; then

$$
\begin{aligned}
\dot{Z}(t) & =N \delta \int_{0}^{\omega} g(\tau) e^{-n \tau} y(t-\tau) d \tau-c v(t)-\frac{q \mu}{r} z(t) \\
& \leq N \delta L_{1} \int_{0}^{\omega} g(\tau) e^{-n \tau} d \tau-\sigma_{2}\left(v(t)+\frac{q}{r} z(t)\right) \\
& =N \delta L_{1} \int_{0}^{\omega} g(\tau) e^{-n \tau} d \tau-\sigma_{2} Z(t) \leq N \delta L_{1}-\sigma_{2} Z(t),
\end{aligned}
$$

where $\sigma_{2}=\min \{c, \mu\}$. Hence, $\limsup _{t \rightarrow \infty} Z(t) \leq L_{2}$, where $L_{2}=N \delta L_{1} / \sigma_{2}$. Since $v(t) \geq 0$, and $v(t) \geq 0$, then $\lim \sup _{t \rightarrow \infty} v(t) \leq L_{2}$ and $\lim \sup _{t \rightarrow \infty} z(t) \leq L_{2}$.

Therefore, $x(t), y(t), v(t)$, and $z(t)$ are ultimately bounded.

2.2. Steady States. We define the basic reproduction number for system (2)-(5) as

$$
R_{0}=\frac{N F G \beta \lambda}{c d}
$$

and the antibody immune response reproduction number

$$
R_{1}=\frac{R_{0}}{1+((\mu \alpha d+\mu \beta) / r d)} .
$$

Clearly $R_{1}<R_{0}$. It can be seen that system (2)-(5) has an uninfected steady state $E_{0}=\left(x_{0}, 0,0,0\right)$, where $x_{0}=\lambda / d$. In addition to $E_{0}$, the system has an infected steady state without immune response $E_{1}\left(x_{1}, y_{1}, v_{1}, 0\right)$ and infected steady state with immune response $E_{2}\left(x_{2}, y_{2}, v_{2}, z_{2}\right)$, where

$$
\begin{aligned}
& x_{1}=\frac{x_{0}\left(1+\alpha v_{1}\right)}{R_{0}}, \quad y_{1}=\frac{c}{N \delta G} v_{1}, \\
& v_{1}=\frac{d}{\alpha d+\beta}\left(R_{0}-1\right), \\
& x_{2}=\frac{\lambda}{d+\left(\beta v_{2} /\left(1+\alpha v_{2}\right)\right)}, \\
& y_{2}=\frac{F \beta \lambda v_{2}}{\delta\left(d\left(1+\alpha v_{2}\right)+\beta v_{2}\right)}, \\
& v_{2}=\frac{\mu}{r}, \quad z_{2}=\frac{c}{q}\left(R_{1}-1\right) .
\end{aligned}
$$

From the above we have the following:

(i) if $R_{0}>1$, then there exists a positive steady state $E_{1}\left(x_{1}, y_{1}, v_{1}, 0\right)$;

(ii) if $R_{1}>1$, then there exists a positive steady state $E_{2}\left(x_{2}, y_{2}, v_{2}, z_{2}\right)$.

\section{Global Stability}

In this section, we prove the global stability of the steady states of system (2)-(5) employing the method of Lyapunov 
functional which is used in [32] for SIR epidemic model with distributed delay. Next we will use the following notation: $b=b(t)$ for any $b \in\{x, y, v, z\}$. We also define a function $H:(0, \infty) \rightarrow[0, \infty)$ as

$$
H(b)=b-1-\ln (b) \text {. }
$$

It is clear that $H(b) \geq 0$ for any $b>0$ and $H$ has the global minimum $H(1)=0$.

Theorem 2. If $R_{0} \leq 1$, then $E_{0}$ is GAS.

Proof. Define a Lyapunov functional $W_{0}$ as follows:

$$
\begin{aligned}
W_{0}=N F G[ & x_{0} H\left(\frac{x}{x_{0}}\right)+\frac{1}{F} y+\frac{\beta}{F} \\
& \times \int_{0}^{h} f(\tau) e^{-m \tau} \\
& \times \int_{0}^{\tau} \frac{x(t-\theta) v(t-\theta)}{1+\alpha v(t-\theta)} d \theta d \tau \\
& \left.+\frac{\delta}{F G} \int_{0}^{\omega} g(\tau) e^{-n \tau} \int_{0}^{\tau} y(t-\theta) d \theta d \tau\right]+v+\frac{q}{r} z .
\end{aligned}
$$

The time derivative of $W_{0}$ along the trajectories of (2)-(5) satisfies

$$
\begin{aligned}
& \frac{d W_{0}}{d t}=N F G\left[\left(1-\frac{x_{0}}{x}\right)\left(\lambda-d x-\frac{\beta x v}{1+\alpha v}\right)\right. \\
& +\frac{\beta}{F} \int_{0}^{h} f(\tau) e^{-m \tau} \frac{x(t-\tau) v(t-\tau)}{1+\alpha v(t-\tau)} d \tau-\frac{\delta}{F} y \\
& +\frac{\beta}{F} \int_{0}^{h} f(\tau) e^{-m \tau} \\
& \times\left(\frac{x v}{1+\alpha v}-\frac{x(t-\tau) v(t-\tau)}{1+\alpha v(t-\tau)}\right) d \tau \\
& \left.+\frac{\delta}{F G} \int_{0}^{\omega} g(\tau) e^{-n \tau}(y-y(t-\tau)) d \tau\right] \\
& +N \delta \int_{0}^{\omega} g(\tau) e^{-n \tau} y(t-\tau) d \tau \\
& -c v-q v z+q v z-\frac{q \mu}{r} z \\
& =N F G\left[-d \frac{\left(x-x_{0}\right)^{2}}{x}+\frac{\beta x_{0} v}{1+\alpha v}\right]-c v-\frac{q \mu}{r} z \\
& =-N F G d \frac{\left(x-x_{0}\right)^{2}}{x}-c v \\
& +c v \frac{R_{0}(1+\alpha v-\alpha v)}{1+\alpha v}-\frac{q \mu}{r} z \\
& =-\frac{N F G d}{x}\left(x-x_{0}\right)^{2}+c v\left(R_{0}-1\right) \\
& -\frac{R_{0} c \alpha v^{2}}{1+\alpha v}-\frac{q \mu}{r} z
\end{aligned}
$$

If $R_{0} \leq 1$, then $d W_{0} / d t \leq 0$ for all $x, v, z>0$. By Theorem 5.3.1 in [33], the solutions of system (2)-(5) limit to $M$, the largest invariant subset of $\left\{d W_{0} / d t=0\right\}$. Clearly, it follows from (17) that $d W_{0} / d t=0$ if and only if $x=x_{0}, v=0$, and $z=0$. Noting that $M$ is invariant for each element of $M$, we have $v=0$, and $z=0$, and then $\dot{v}=0$. From (4) we derive that

$$
0=\dot{v}=N \delta \int_{0}^{\omega} g(\tau) e^{-n \tau} y(t-\tau) d \tau
$$

This yields $y=0$. Hence, $d W_{0} / d t=0$ if and only if $x=x_{0}$, $y=0, v=0$, and $z=0$. From LaSalle's Invariance Principle, $E_{0}$ is GAS.

Theorem 3. If $R_{1} \leq 1<R_{0}$, then $E_{1}$ is GAS.

Proof. We construct the following Lyapunov functional:

$$
\begin{aligned}
W_{1}=N F G[ & x_{1} H\left(\frac{x}{x_{1}}\right)+\frac{1}{F} y_{1} H\left(\frac{y}{y_{1}}\right) \\
& +\frac{1}{F} \frac{\beta x_{1} v_{1}}{\left(1+\alpha v_{1}\right)} \\
& \times \int_{0}^{h} f(\tau) e^{-m \tau} \\
& \times \int_{0}^{\tau} H\left(\frac{x(t-\theta) v(t-\theta)\left(1+\alpha v_{1}\right)}{x_{1} v_{1}(1+\alpha v(t-\theta))}\right) d \theta d \tau \\
& \left.+\frac{\delta y_{1}}{F G} \int_{0}^{\omega} g(\tau) e^{-n \tau} \int_{0}^{\tau} H\left(\frac{y(t-\theta)}{y_{1}}\right) d \theta d \tau\right] \\
+ & v_{1} H\left(\frac{v}{v_{1}}\right)+\frac{q}{r} z .
\end{aligned}
$$

The time derivative of $W_{1}$ along the trajectories of (2)-(5) is given by

$$
\begin{aligned}
\frac{d W_{1}}{d t}=N F G & {\left[\left(1-\frac{x_{1}}{x}\right)\right.} \\
& \times\left(\lambda-d x-\frac{\beta x v}{1+\alpha v}\right)+\frac{1}{F}\left(1-\frac{y_{1}}{y}\right) \\
& \times\left(\beta \int_{0}^{h} f(\tau) e^{-m \tau} \frac{x(t-\tau) v(t-\tau)}{1+\alpha v(t-\tau)} d \tau-\delta y\right) \\
+ & \frac{\beta}{F} \int_{0}^{h} f(\tau) e^{-m \tau} \\
& \times\left(\frac{x v}{1+\alpha v}-\frac{x(t-\tau) v(t-\tau)}{1+\alpha v(t-\tau)}+\frac{x_{1} v_{1}}{1+\alpha v_{1}}\right. \\
& \left.\times \ln \left(\frac{x(t-\tau) v(t-\tau)(1+\alpha v)}{x v(1+\alpha v(t-\tau))}\right)\right) d \tau
\end{aligned}
$$




$$
\begin{aligned}
& +\frac{\delta}{F G} \int_{0}^{\omega} g(\tau) e^{-n \tau} \\
& \left.\quad \times\left(y-y(t-\tau)+y_{1} \ln \left(\frac{y(t-\tau)}{y}\right)\right) d \tau\right] \\
& +\left(1-\frac{v_{1}}{v}\right)\left(N \delta \int_{0}^{\omega} g(\tau) e^{-n \tau} y(t-\tau) d \tau\right. \\
& -c v-q z v)+q v z-\frac{q \mu}{r} z .
\end{aligned}
$$

Using the steady state conditions for $E_{1}$ :

$$
\begin{gathered}
\lambda=d x_{1}+\frac{\beta x_{1} v_{1}}{1+\alpha v_{1}}, \\
\delta y_{1}=F \frac{\beta x_{1} v_{1}}{1+\alpha v_{1}}, \\
c v_{1}=N \delta G y_{1},
\end{gathered}
$$

we have

$$
\begin{aligned}
\frac{d W_{1}}{d t}=N F G[ & -d \frac{\left(x-x_{1}\right)^{2}}{x}+\frac{\beta x_{1} v_{1}}{1+\alpha v_{1}} \\
& -\frac{\beta x_{1} v_{1}}{1+\alpha v_{1}} \frac{x_{1}}{x}+\frac{\beta x_{1} v}{1+\alpha v}-\frac{1}{F} \frac{\beta x_{1} v_{1}}{\left(1+\alpha v_{1}\right)} \\
& \times \int_{0}^{h} f(\tau) e^{-m \tau} \\
& \times \frac{y_{1} x(t-\tau) v(t-\tau)\left(1+\alpha v_{1}\right)}{y x_{1} v_{1}(1+\alpha v(t-\tau))} d \tau \\
+ & \frac{\delta}{F} y_{1}+\frac{1}{F} \frac{\beta x_{1} v_{1}}{1+\alpha v_{1}} \\
+ & \times \int_{0}^{h} f(\tau) e^{-m \tau} \int_{0}^{\omega} g(\tau) e^{-n \tau} \frac{v_{1} y(t-\tau)}{v y_{1}} d \tau \\
+ & \left.\frac{\delta y_{1}}{F G} \int_{0}^{\omega} g(\tau) e^{-n \tau} \ln \left(\frac{y(t-\tau)}{y}\right) d \tau\right] d \tau \\
& \times \ln \left(\frac{x(t-\tau) v(t-\tau)(1+\alpha v)}{x v(1+\alpha v(t-\tau))}\right) d
\end{aligned}
$$

Using the following equalities:

$$
\begin{gathered}
\ln \left(\frac{x(t-\tau) v(t-\tau)(1+\alpha v)}{x v(1+\alpha v(t-\tau))}\right) \\
=\ln \left(\frac{y_{1} x(t-\tau) v(t-\tau)\left(1+\alpha v_{1}\right)}{y x_{1} v_{1}(1+\alpha v(t-\tau))}\right) \\
\quad+\ln \left(\frac{x_{1}}{x}\right)+\ln \left(\frac{v_{1} y}{v y_{1}}\right)+\ln \left(\frac{1+\alpha v}{1+\alpha v_{1}}\right), \\
\ln \left(\frac{y(t-\tau)}{y}\right)=\ln \left(\frac{v y_{1}}{v_{1} y}\right)+\ln \left(\frac{v_{1} y(t-\tau)}{v y_{1}}\right),
\end{gathered}
$$

then collecting terms of (22), we obtain

$$
\begin{aligned}
& \frac{d W_{1}}{d t}=N F G\left[-d \frac{\left(x-x_{1}\right)^{2}}{x}-\frac{\delta y_{1}}{F}\left(\frac{x_{1}}{x}-1-\ln \frac{x_{1}}{x}\right)\right. \\
& +\frac{\delta y_{1}}{F} \frac{v\left(1+\alpha v_{1}\right)}{v_{1}(1+\alpha v)}-\frac{\delta y_{1}}{F^{2}} \\
& \times \int_{0}^{h} f(\tau) e^{-m \tau} \\
& \times\left(\frac{y_{1} x(t-\tau) v(t-\tau)\left(1+\alpha v_{1}\right)}{y x_{1} v_{1}(1+\alpha v(t-\tau))}-1\right. \\
& \left.-\ln \left(\frac{y_{1} x(t-\tau) v(t-\tau)\left(1+\alpha v_{1}\right)}{y x_{1} v_{1}(1+\alpha v(t-\tau))}\right)\right) d \tau \\
& -\frac{\delta y_{1}}{F G} \int_{0}^{w} g(\tau) e^{-n \tau} \\
& \times\left(\frac{v_{1} y(t-\tau)}{v y_{1}}-1\right. \\
& \left.-\ln \left(\frac{v_{1} y(t-\tau)}{v y_{1}}\right)\right) d \tau \\
& \left.+\frac{\delta y_{1}}{F}\left(\ln \left(\frac{1+\alpha v}{1+\alpha v_{1}}\right)-\frac{v}{v_{1}}\right)\right] \\
& +q z\left(v_{1}-\frac{\mu}{r}\right) \\
& =N F G\left[-d \frac{\left(x-x_{1}\right)^{2}}{x}+\frac{\delta y_{1}}{F}\right. \\
& \times\left(-1+\frac{v\left(1+\alpha v_{1}\right)}{v_{1}(1+\alpha v)}-\frac{v}{v_{1}}+\frac{1+\alpha v}{1+\alpha v_{1}}\right) \\
& -\frac{\delta y_{1}}{F} H\left(\frac{x_{1}}{x}\right)-\frac{\delta y_{1}}{F^{2}}
\end{aligned}
$$




$$
\begin{aligned}
& \times \int_{0}^{h} f(\tau) e^{-m \tau} \\
& \times H\left(\frac{y_{1} x(t-\tau) v(t-\tau)\left(1+\alpha v_{1}\right)}{y x_{1} v_{1}(1+\alpha v(t-\tau))}\right) d \tau \\
& -\frac{\delta}{F G} y_{1} \int_{0}^{\omega} g(\tau) e^{-n \tau} H\left(\frac{v_{1} y(t-\tau)}{v y_{1}}\right) d \tau \\
& \left.-\frac{\delta y_{1}}{F} H\left(\frac{1+\alpha v}{1+\alpha v_{1}}\right)\right]+q z\left(v_{1}-\frac{\mu}{r}\right) \\
& =N F G\left[-d \frac{\left(x-x_{1}\right)^{2}}{x}-\frac{\delta y_{1}}{F} \frac{\alpha\left(v-v_{1}\right)^{2}}{v_{1}(1+\alpha v)\left(1+\alpha v_{1}\right)}\right. \\
& -\frac{\delta y_{1}}{F} H\left(\frac{x_{1}}{x}\right)-\frac{\delta y_{1}}{F^{2}} \\
& \times \int_{0}^{h} f(\tau) e^{-m \tau} \\
& \times H\left(\frac{y_{1} x(t-\tau) v(t-\tau)\left(1+\alpha v_{1}\right)}{y x_{1} v_{1}(1+\alpha v(t-\tau))}\right) d \tau \\
& -\frac{\delta}{F G} y_{1} \int_{0}^{\omega} g(\tau) e^{-n \tau} H\left(\frac{v_{1} y(t-\tau)}{v y_{1}}\right) d \tau \\
& \left.-\frac{\delta y_{1}}{F} H\left(\frac{1+\alpha v}{1+\alpha v_{1}}\right)\right] \\
& +\frac{q d}{\alpha d+\beta}\left(1+\frac{\mu \alpha d+\mu \beta}{r d}\right)\left(R_{1}-1\right) z \text {. }
\end{aligned}
$$

Thus, if $R_{0}>1$, then $x_{1}, y_{1}$, and $v_{1}>0$, and hence, if $R_{1} \leq 1$, then $d W_{1} / d t \leq 0$ for all $x, y, v>0$. By Theorem 5.3.1 in [33], the solutions of system (2)-(5) limit to $M$, the largest invariant subset of $\left\{d W_{1} / d t=0\right\}$. It can be seen that $d W_{1} / d t=0$ if and only if $x=x_{1}, v=v_{1}$, and $H=0$; that is,

$$
\frac{y_{1} x(t-\tau) v(t-\tau)\left(1+\alpha v_{1}\right)}{y x_{1} v_{1}(1+\alpha v(t-\tau))}=\frac{v_{1} y(t-\tau)}{v y_{1}}=1
$$

$$
\text { for almost all } \tau \in[0, \rho] \text {. }
$$

From (25), if $v=v_{1}$, then $y=y_{1}$, and hence $d W_{1} / d t$ equals zero at $E_{1}$. LaSalle's Invariance Principle implies global stability of $E_{1}$.

Theorem 4. If $R_{1}>1$, then $E_{2}$ is GAS.

Proof. We construct the following Lyapunov functional:

$$
\begin{gathered}
W_{2}=N F G\left[x_{2} H\left(\frac{x}{x_{2}}\right)+\frac{1}{F} y_{2} H\left(\frac{y}{y_{2}}\right)\right. \\
+\frac{1}{F} \frac{\beta x_{2} v_{2}}{\left(1+\alpha v_{2}\right)}
\end{gathered}
$$

$$
\begin{aligned}
& \times \int_{0}^{h} f(\tau) e^{-m \tau} \\
& \quad \times \int_{0}^{\tau} H\left(\frac{x(t-\theta) v(t-\theta)\left(1+\alpha v_{2}\right)}{x_{2} v_{2}(1+\alpha v(t-\theta))}\right) d \theta d \tau \\
& \left.+\frac{\delta y_{2}}{F G} \int_{0}^{\omega} g(\tau) e^{-n \tau} \int_{0}^{\tau} H\left(\frac{y(t-\theta)}{y_{2}}\right) d \theta d \tau\right] \\
& +v_{2} H\left(\frac{v}{v_{2}}\right)+\frac{q}{r} z_{2} H\left(\frac{z}{z_{2}}\right) .
\end{aligned}
$$

The time derivative of $W_{2}$ along the trajectories of (2)-(5) is given by

$$
\begin{aligned}
& \frac{d W_{2}}{d t}=N F G\left[\left(1-\frac{x_{2}}{x}\right)\left(\lambda-d x-\frac{\beta x v}{1+\alpha v}\right)+\frac{1}{F}\left(1-\frac{y_{2}}{y}\right)\right. \\
& \times\left(\beta \int_{0}^{h} f(\tau) e^{-m \tau} \frac{x(t-\tau) v(t-\tau)}{1+\alpha v(t-\tau)} d \tau-\delta y\right) \\
& +\frac{\beta}{F} \int_{0}^{h} f(\tau) e^{-m \tau} \\
& \times\left(\frac{x v}{1+\alpha v}-\frac{x(t-\tau) v(t-\tau)}{1+\alpha v(t-\tau)}+\frac{x_{2} v_{2}}{1+\alpha v_{2}}\right. \\
& \left.\times \ln \left(\frac{x(t-\tau) v(t-\tau)(1+\alpha v)}{x v(1+\alpha v(t-\tau))}\right)\right) d \tau \\
& +\frac{\delta}{F G} \int_{0}^{\omega} g(\tau) e^{-n \tau} \\
& \times(y-y(t-\tau) \\
& \left.\left.+y_{2} \ln \left(\frac{y(t-\tau)}{y}\right)\right) d \tau\right]+\left(1-\frac{v_{2}}{v}\right) \\
& \times\left(N \delta \int_{0}^{\omega} g(\tau) e^{-n \tau} y(t-\tau) d \tau-c v-q z v\right) \\
& +\left(1-\frac{z_{2}}{z}\right)\left(q v z-\frac{q \mu}{r} z\right) .
\end{aligned}
$$

Using the steady state conditions for $E_{2}$ :

$$
\begin{aligned}
\lambda & =d x_{2}+\beta \frac{x_{2} v_{2}}{1+\alpha v_{2}}, \\
c v_{2} & =N F G\left(\frac{\delta}{F} y_{2}\right)-q v_{2} z_{2}, \\
\mu & =r v_{2}
\end{aligned}
$$


we obtain

$$
\begin{aligned}
\frac{d W_{2}}{d t}=N F G[ & -d \frac{\left(x-x_{2}\right)^{2}}{x}+\frac{\beta x_{2} v_{2}}{1+\alpha v_{2}} \\
& -\frac{\beta x_{2} v_{2}}{1+\alpha v_{2}} \frac{x_{2}}{x}+\frac{\beta x_{2} v}{1+\alpha v}-\frac{1}{F} \frac{\beta x_{2} v_{2}}{1+\alpha v_{2}} \\
& \times \int_{0}^{h} f(\tau) e^{-m \tau} \\
& \times \frac{y_{2} x(t-\tau) v(t-\tau)\left(1+\alpha v_{2}\right)}{y x_{2} v_{2}(1+\alpha v(t-\tau))} d \tau \\
& +\frac{\delta}{F} y_{2}+\frac{1}{F} \frac{\beta x_{2} v_{2}}{1+\alpha v_{2}} \\
& \times \int_{0}^{h} f(\tau) e^{-m \tau} \\
& \times \ln \left(\frac{x(t-\tau) v(t-\tau)(1+\alpha v)}{x v(1+\alpha v(t-\tau))}\right) d \tau \\
+ & \left.\frac{\delta y_{2}}{F G} \int_{0}^{\omega} g(\tau) e^{-n \tau} \ln \left(\frac{y(t-\tau)}{y}\right) d \tau\right] \\
- & N \delta y_{2} \int_{0}^{\omega} g(\tau) e^{-n \tau} \frac{v_{2} y(t-\tau)}{v y_{2}} d \tau-c v \\
+ & +q v_{2}+q v_{2} z-q v z_{2}-\frac{q \mu}{r} z+\frac{q \mu}{r} z_{2} .
\end{aligned}
$$

Using the following equalities:

$$
\begin{gathered}
c v=c v_{2} \frac{v}{v_{2}}=N F G\left(\frac{\delta}{F} y_{2} \frac{v}{v_{2}}\right)-q v z_{2}, \\
\ln \left(\frac{x(t-\tau) v(t-\tau)(1+\alpha v)}{x v(1+\alpha v(t-\tau))}\right) \\
=\ln \left(\frac{y_{2} x(t-\tau) v(t-\tau)\left(1+\alpha v_{2}\right)}{y x_{2} v_{2}(1+\alpha v(t-\tau))}\right) \\
+\ln \left(\frac{x_{2}}{x}\right)+\ln \left(\frac{v_{2} y}{v y_{2}}\right)+\ln \left(\frac{1+\alpha v}{1+\alpha v_{2}}\right), \\
\ln \left(\frac{y(t-\tau)}{y}\right)=\ln \left(\frac{v y_{2}}{v_{2} y}\right)+\ln \left(\frac{v_{2} y(t-\tau)}{v y_{2}}\right),
\end{gathered}
$$

and collecting terms of (29), we obtain

$$
\begin{aligned}
\frac{d W_{2}}{d t}=N F G[ & -d \frac{\left(x-x_{2}\right)^{2}}{x}-\frac{\delta y_{1}}{F}\left(\frac{x_{2}}{x}-1-\ln \frac{x_{2}}{x}\right) \\
& +\frac{\delta y_{2}}{F} \frac{v\left(1+\alpha v_{2}\right)}{v_{2}(1+\alpha v)}-\frac{\delta y_{2}}{F^{2}}
\end{aligned}
$$

$$
\begin{aligned}
& \times \int_{0}^{h} f(\tau) e^{-m \tau} \\
& \times\left(\frac{y_{2} x(t-\tau) v(t-\tau)\left(1+\alpha v_{2}\right)}{y x_{2} v_{2}(1+\alpha v(t-\tau))}-1\right. \\
& \left.-\ln \left(\frac{y_{2} x(t-\tau) v(t-\tau)\left(1+\alpha v_{2}\right)}{y x_{2} v_{2}(1+\alpha v(t-\tau))}\right)\right) d \tau \\
& -\frac{\delta y_{2}}{F G} \int_{0}^{\omega} g(\tau) e^{-n \tau}\left(\frac{v_{2} y(t-\tau)}{v y_{2}}-1\right. \\
& \left.-\ln \left(\frac{v_{2} y(t-\tau)}{v y_{2}}\right)\right) d \tau \\
& \left.+\frac{\delta y_{2}}{F}\left(\ln \left(\frac{1+\alpha v}{1+\alpha v_{2}}\right)-\frac{v}{v_{2}}\right)\right] \\
& =N F G\left[-d \frac{\left(x-x_{2}\right)^{2}}{x}-\frac{\delta y_{2}}{F} \frac{\alpha\left(v-v_{2}\right)^{2}}{v_{2}(1+\alpha v)\left(1+\alpha v_{2}\right)}\right. \\
& -\frac{\delta y_{2}}{F} H\left(\frac{x_{2}}{x}\right)-\frac{\delta y_{2}}{F^{2}} \\
& \times \int_{0}^{h} f(\tau) e^{-m \tau} \\
& \times H\left(\frac{y_{2} x(t-\tau) v(t-\tau)\left(1+\alpha v_{2}\right)}{y x_{2} v_{2}(1+\alpha v(t-\tau))}\right) d \tau \\
& -\frac{\delta}{F G} y_{2} \int_{0}^{\omega} g(\tau) e^{-n \tau} H\left(\frac{v_{2} y(t-\tau)}{v y_{2}}\right) d \tau \\
& \left.-\frac{\delta y_{2}}{F} H\left(\frac{1+\alpha v}{1+\alpha v_{2}}\right)\right] \text {. }
\end{aligned}
$$

Thus, if $R_{1}>1$, then $x_{2}, y_{2}, v_{2}$, and $z_{2}>0$ and $d W_{2} / d t \leq 0$. By Theorem 5.3.1 in [33], the solutions of system (2)-(5) limit to $M$, the largest invariant subset of $\left\{d W_{2} / d t=0\right\}$. It can be seen that $d W_{2} / d t=0$ if and only if $x=x_{2}, v=v_{2}$, and $H=0$; that is,

$$
\frac{y_{2} x(t-\tau) v(t-\tau)\left(1+\alpha v_{2}\right)}{y x_{2} v_{2}(1+\alpha v(t-\tau))}=\frac{v_{2} y(t-\tau)}{v y_{2}}=\frac{1+\alpha v}{1+\alpha v_{2}}=1
$$$$
\text { for almost all } \tau \in[0, \rho] \text {. }
$$

Then from (32) $y=y_{2}$, and hence $d W_{2} / d t=0$ at the steady state $E_{2}$. LaSalle's Invariance Principle implies global stability of $E_{2}$.

\section{Conclusion}

In this paper, we have proposed a virus infection model which describes the interaction of the virus with the uninfected and infected cells taking into account the antibody immune response. The infection rate is given by saturation functional response. Two types of distributed time delays have been 
incorporated into the model to describe the time needed for infection of uninfected cell and virus replication. Using the method of Lyapunov functional, we have established that the global dynamics of the model is determined by two threshold parameters $R_{0}$ and $R_{1}$. The basic reproduction number viral infection $R_{0}$ determines whether a chronic infection can be established, and the antibody immune response reproduction number $R_{1}$ determines whether a persistent antibody immune response can be established. We have proven that if $R_{0} \leq 1$, then the uninfected steady state is GAS, and the viruses are cleared. If $R_{1} \leq 1<R_{0}$, then the infected steady state without antibody immune response is GAS, and the infection becomes chronic but without persistent antibody immune response. If $R_{1}>1$, then the infected steady state with antibody immune response is GAS, and the infection is chronic with persistent antibody immune response. We note that the effect of the time delay appears in the parameters $F$ and $G$. Since $0<F \leq 1$ and $0<G \leq 1$, then the intracellular delay can reduce the parameters $R_{0}$ and $R_{1}$. As a consequence, ignoring the delay will produce overestimation of $R_{0}$.

\section{Conflict of Interests}

The authors declare that there is no conflict of interests regarding the publication of this paper.

\section{Acknowledgments}

This article was funded by the Deanship of Scientific Research (DSR), King Abdulaziz University, Jeddah, Saudi Arabia. The authors, therefore, acknowledge with thanks DSR technical and financial support. The authors are also grateful to Professor Victor Kozyakin and to the anonymous reviewers for constructive suggestions and valuable comments, which improved the quality of the article.

\section{References}

[1] M. A. Nowak and R. M. May, Virus Dynamicss: Mathematical Principles of Immunology and Virology, Oxford University Press, Oxford, UK, 2000.

[2] A. S. Perelson and P. W. Nelson, "Mathematical analysis of HIV1 dynamics in vivo," SIAM Review, vol. 41, no. 1, pp. 3-44, 1999.

[3] D. S. Callaway and A. S. Perelson, "HIV-1 infection and low steady state viral loads," Bulletin of Mathematical Biology, vol. 64, no. 1, pp. 29-64, 2002.

[4] P. K. Roy, A. N. Chatterjee, D. Greenhalgh, and Q. J. A. Khan, "Long term dynamics in a mathematical model of HIV1 infection with delay in different variants of the basic drug therapy model," Nonlinear Analysis: Real World Applications, vol. 14, no. 3, pp. 1621-1633, 2013.

[5] P. W. Nelson, J. D. Murray, and A. S. Perelson, "A model of HIV-1 pathogenesis that includes an intracellular delay," Mathematical Biosciences, vol. 163, no. 2, pp. 201-215, 2000.

[6] P. W. Nelson and A. S. Perelson, "Mathematical analysis of delay differential equation models of HIV-1 infection," Mathematical Biosciences, vol. 179, no. 1, pp. 73-94, 2002.
[7] R. V. Culshaw and S. Ruan, "A delay-differential equation model of HIV infection of $\mathrm{CD}^{+}$T-cells," Mathematical Biosciences, vol. 165, no. 1, pp. 27-39, 2000.

[8] J. E. Mittler, B. Sulzer, A. U. Neumann, and A. S. Perelson, "Influence of delayed viral production on viral dynamics in HIV-1 infected patients," Mathematical Biosciences, vol. 152, no. 2, pp. 143-163, 1998.

[9] A. M. Elaiw and A. S. Alsheri, "Global Dynamics of HIV Infection of CD $4{ }^{+}$T Cells and Macrophages," Discrete Dynamics in Nature and Society, vol. 2013, Article ID 264759, 8 pages, 2013.

[10] A. M. Elaiw and S. A. Azoz, "Global properties of a class of HIV infection models with Beddington-DeAngelis functional response," Mathematical Methods in the Applied Sciences, vol. 36, no. 4, pp. 383-394, 2013.

[11] A. Elaiw, I. Hassanien, and S. Azoz, "Global stability of HIV infection models with intracellular delays," Journal of the Korean Mathematical Society, vol. 49, no. 4, pp. 779-794, 2012.

[12] A. M. Elaiw and M. A. Alghamdi, "Global properties of virus dynamics models with multitarget cells and discrete-time delays," Discrete Dynamics in Nature and Society, vol. 2011, Article ID 201274, 19 pages, 2011.

[13] A. M. Elaiw, "Global properties of a class of virus infection models with multitarget cells," Nonlinear Dynamics, vol. 69, no. 1-2, pp. 423-435, 2012.

[14] A. M. Elaiw, "Global dynamics of an HIV infection model with two classes of target cells and distributed delays," Discrete Dynamics in Nature and Society, vol. 2012, Article ID 253703, 13 pages, 2012.

[15] M. A. Nowak and C. R. M. Bangham, "Population dynamics of immune responses to persistent viruses," Science, vol. 272, no. 5258, pp. 74-79, 1996.

[16] K. Wang, A. Fan, and A. Torres, "Global properties of an improved hepatitis B virus model," Nonlinear Analysis: Real World Applications, vol. 11, no. 4, pp. 3131-3138, 2010.

[17] S. Zeuzem, J. M. Schmidt, J.-H. Lee, B. Rüster, and W. K. Roth, "Effect of interferon alfa on the dynamics of hepatitis $\mathrm{C}$ virus turnover in vivo," Hepatology, vol. 23, no. 2, pp. 366-371, 1996.

[18] A. U. Neumann, N. P. Lam, H. Dahari et al., "Hepatitis C viral dynamics in vivo and the antiviral efficacy of interferon$\alpha$ therapy," Science, vol. 282, no. 5386, pp. 103-107, 1998.

[19] J. A. Deans and S. Cohen, "Immunology of malaria," Annual Review of Microbiology, vol. 37, pp. 25-49, 1983.

[20] R. M. Anderson, R. M. May, and S. Gupta, "Non-linear phenomena in host-parasite interactions," Parasitology, vol. 99, pp. S59-S79, 1989.

[21] A. Murase, T. Sasaki, and T. Kajiwara, "Stability analysis of pathogen-immune interaction dynamics," Journal of Mathematical Biology, vol. 51, no. 3, pp. 247-267, 2005.

[22] D. Wodarz, R. M. May, and M. A. Nowak, "The role of antigenindependent persistence of memory cytotoxic T lymphocytes," International Immunology, vol. 12, no. 4, pp. 467-477, 2000.

[23] C. Chiyaka, W. Garira, and S. Dube, "Modelling immune response and drug therapy in human malaria infection," Computational and Mathematical Methods in Medicine, vol. 9, no. 2, pp. 143-163, 2008.

[24] A. S. Perelson, "Modelling viral and immune system dynamics," Nature Reviews Immunology, vol. 2, no. 1, pp. 28-36, 2002.

[25] S. Wang and D. Zou, "Global stability of in-host viral models with humoral immunity and intracellular delays," Applied Mathematical Modelling, vol. 36, no. 3, pp. 1313-1322, 2012. 
[26] H. F. Huo, Y. L. Tang, and L. X. Feng, "A virus dynamics model with saturation infection and humoral immunity," International Journal of Mathematical Analysis, vol. 6, no. 40, pp. 1977-1983, 2012.

[27] N. M. Dixit and A. S. Perelson, "Complex patterns of viral load decay under antiretroviral therapy: influence of pharmacokinetics and intracellular delay," Journal of Theoretical Biology, vol. 226, no. 1, pp. 95-109, 2004.

[28] X. Wang and S. Liu, "A class of delayed viral models with saturation infection rate and immune response," Mathematical Methods in the Applied Sciences, vol. 36, no. 2, pp. 125-142, 2013.

[29] A. Korobeinikov, "Global properties of infectious disease models with nonlinear incidence," Bulletin of Mathematical Biology, vol. 69, no. 6, pp. 1871-1886, 2007.

[30] G. Huang, Y. Takeuchi, and W. Ma, "Lyapunov functionals for delay differential equations model of viral infections," SIAM Journal on Applied Mathematics, vol. 70, no. 7, pp. 2693-2708, 2010.

[31] X. Song and A. U. Neumann, "Global stability and periodic solution of the viral dynamics," Journal of Mathematical Analysis and Applications, vol. 329, no. 1, pp. 281-297, 2007.

[32] C. C. McCluskey, "Complete global stability for an SIR epidemic model with delay-distributed or discrete," Nonlinear Analysis: Real World Applications, vol. 11, no. 1, pp. 55-59, 2010.

[33] J. K. Hale and S. M. Verduyn Lunel, Introduction to FunctionalDifferential Equations, vol. 99, Springer, New York, NY, USA, 1993. 


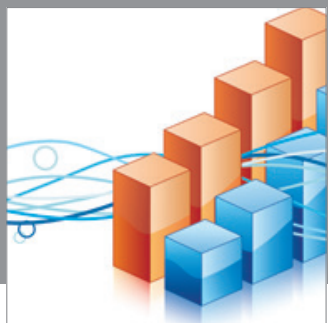

Advances in

Operations Research

mansans

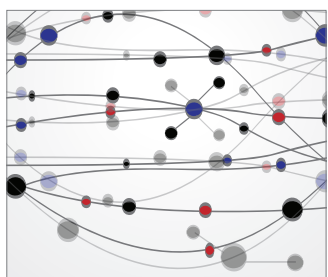

The Scientific World Journal
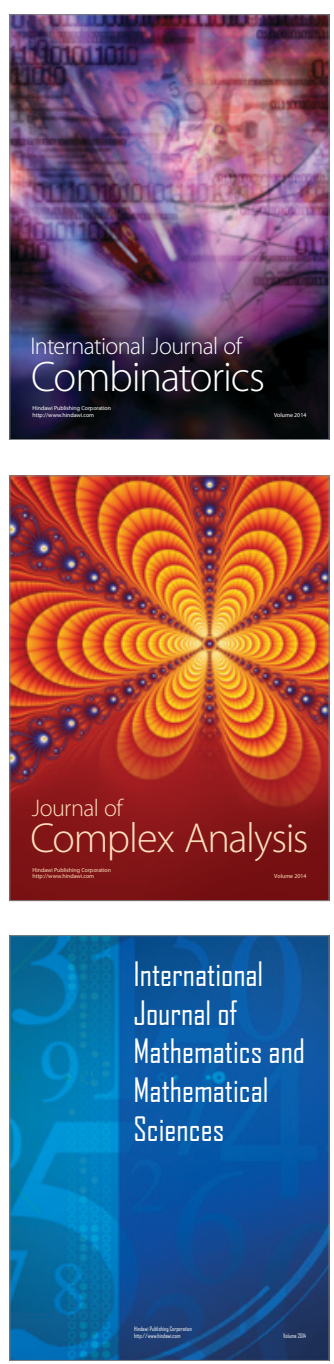
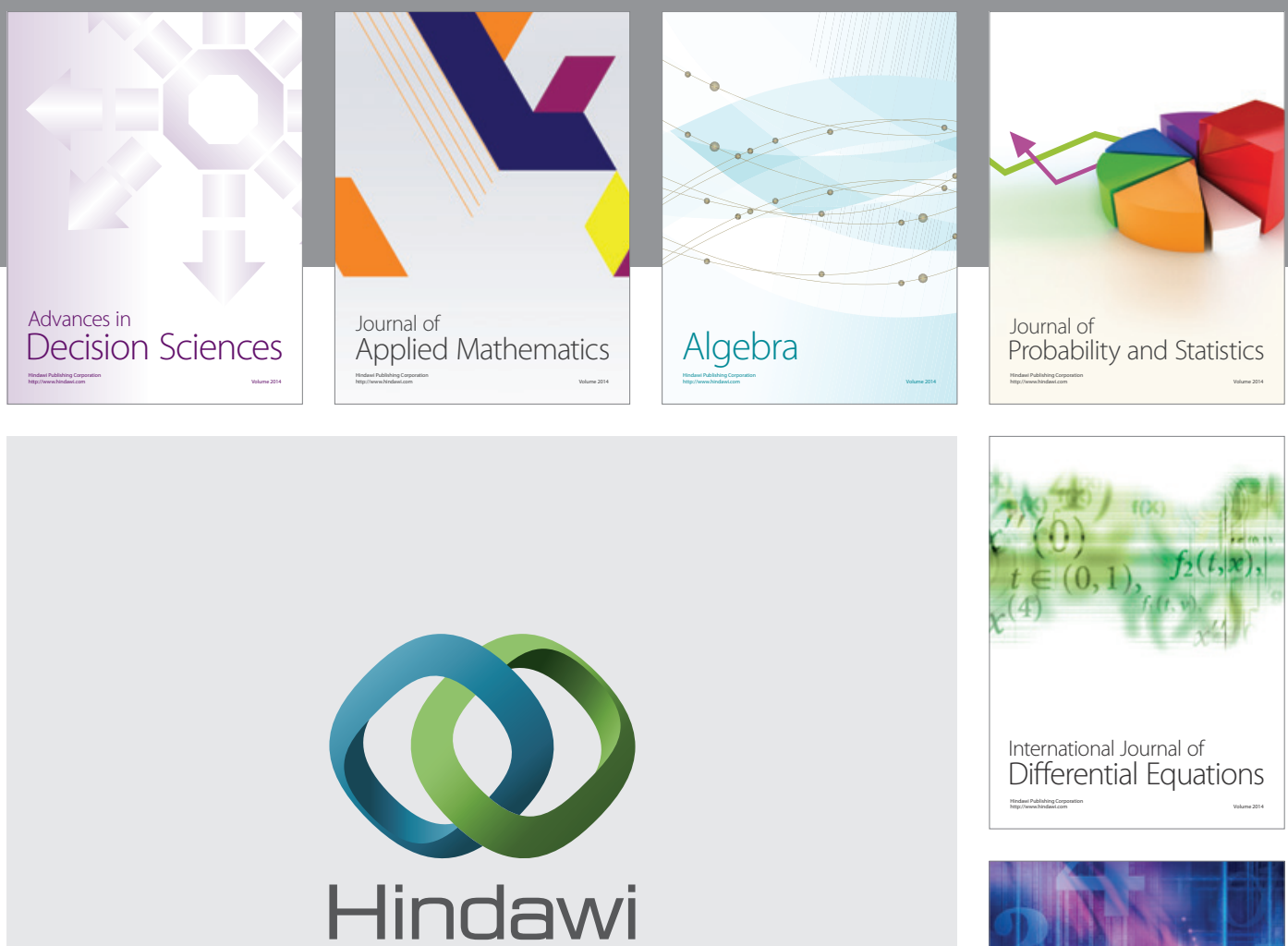

Submit your manuscripts at http://www.hindawi.com
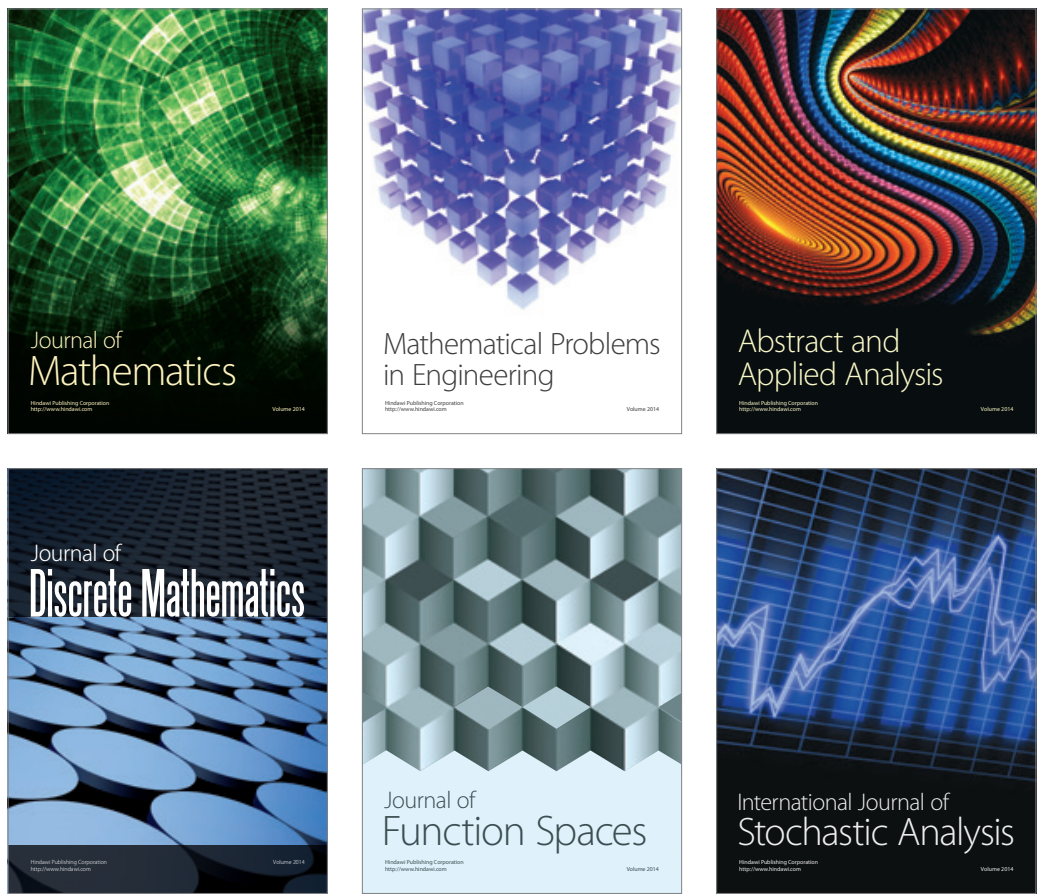

Journal of

Function Spaces

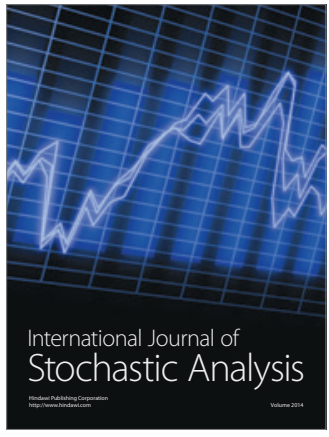

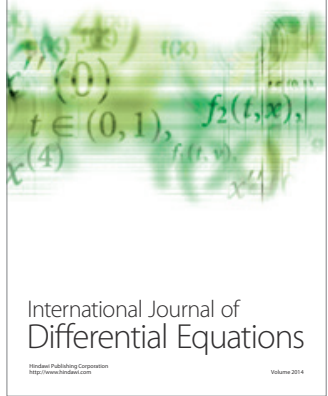
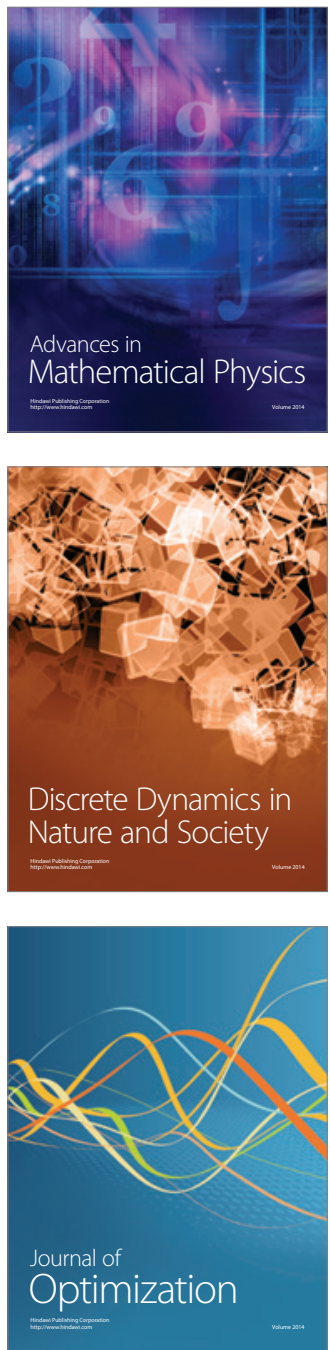